\title{
Receding Horizon Control of Tether Systems Based on Indirect Legendre Pseudospectral Method
}

\author{
Guoyong $\mathrm{Li}^{1,3}$, Changhou $\mathrm{Lu}^{1,2}{ }^{*}$, Wei Pan ${ }^{1,2}$ and Qing wang ${ }^{1}$ \\ ${ }^{1}$ School of Mechanical Engineering, Shandong University, Jinan, \\ P.R. China, 250061 \\ ${ }^{2}$ Key Laboratory of High-efficiency and Clean Mechanical Manufacture \\ (Shandong University), Ministry of Education, P. R. China \\ ${ }^{3}$ National Network Software Testing Center (Jinan) \\ guoyongli@163.com
}

\begin{abstract}
For controlling the deployment of subsatellite, the dumbbell model of tethered satellite system (TSS) is built. The paper develops a nonlinear receding horizon feedback control rule based on the nonlinear dynamics model using an indirect Legendre psuedospectral method. The nonlinear control problem is approximated with successive linear problems obtained via a quasilinearisation around reference trajectory. The feedback gains of receding horizon control could be obtained via solving a two-point boundary value problem which may be discretized into a set of linear algebraic equations by the indirect Legendre pseudospectral method. A numerical example is included to demonstrate the strong stability and high accuracy of the feedback controller under initial perturbations.
\end{abstract}

Keywords: Tethered satellite; Legendre Pseudospectral Method; Nonlinear; Receding horizon control

\section{Introduction}

The tethered Satellite System is a composite unit composed of two or more satellites connected by a tether [1], which possesses many advantages over other space technologies. It has been proposed for a wide variety of applications in many fields including tether-assisted re-entry, air braking, and electrodynamic drives [1, 2]. In recent years, it has become a research hotspot in the field of space [3, 4]. For a tether application to be successful, the ability to safely deploy a sub-satellite from a spaceship is critical, thus the nonlinear nature of the dynamics of the TSS must be taken into account [5]. Controlling the deployment process has been a significant challenging task with complex constraints. The development of advanced control technologies to meet the demands of this problem has come at a significant cost.

Steindl studied optimal control problems of two degrees of freedom for tethered satellites in deployment. In this study, they designed the optimal open-loop control rule using the Maximum Principle to achieve a controlled the deployment [6]. Gläßel presented an adaptive neural network to control the deployment of a tether-assisted re-entry mission with two degrees of freedom [7]. Williams developed a deployment control strategy for the YES2 mission. The open-loop optimal trajectories were determined by direct pseudospectral method. The linear closed-loop controllers were designed using a receding horizon control

* Changhou Lu: Corresponding author 
that tracked the optimal trajectory [8]. Yan proposed an inner feedback loop of a two-degreeof-freedom control system based on the Legendre Pseudospectral method. By the control method, the control system was reduced to a system of linear equations and could be implemented easily in real time [9]. Ohtsuka presented a receding horizon control strategy for a nonlinear time-varying system based on the stabilized continuation method. This method relied on the explicit integration of states and co-states derived from the calculus of variations [10]. Lu proposed a closed-form approximate receding horizon control law for time-varying systems. In his approach Lu employed the Euler-Simpson approximations for the integral cost and the state derivatives, then solved the quadratic programming problem to obtain the control law [11]. Jin, DP proposed a nonlinear optimal control strategy for the tethered subsatellite system based on quasilinearisation and Chebyshev series [12]. Wen $\mathrm{H}$ presented a solution to the nonlinear optimal feedback control problem for the deployment process of a sub-satellite system using only tether tension. The control scheme used a rapid recomputation of the open-loop optimal control based on a direct Legrendre pseudospectral method [13].

In this article, we present the nonlinear optimal feedback control for the deployment of a tether sub-satellite under complex nonlinear constraints. The nonlinearities in the system model and the constraints of states and control are taken into consideration. The open-loop optimal control can be detemined without any iteration, because the system dynamics and control are discretized with a direct Legendre pseudospectral method. This solution is regarded as the reference trajectory that be traced in close-loop control. The nonlinear receding horizon control law is generated by continuously solving the optimal solution over a finite horizon in real time based on the open-loop optimal control and the indirect Legendre pseudospectral method. Finally, a numerical example is included to demonstrate the strong stability and high accuracy of the feedback controller with the modeling errors and initial states perturbations.

\section{Mathematical Model}

As shown in Figure 1, the left is the whole system map, the right is a detail view that the mother satellite S1 and the sub-satellite S2 are connected via a straight tether. In order to reduce the complexity of the system without losing generality, the elasticity and mass of the tether in the dynamic model is not taken into account. The system could be regarded as two lumped mass which are connected by a rigid, inelastic, massless rod. This simplification, which serves to enhance the computational efficiency, is often called a dumbbell method [3].This mathematical model has often been used to design controllers and verify their performance.
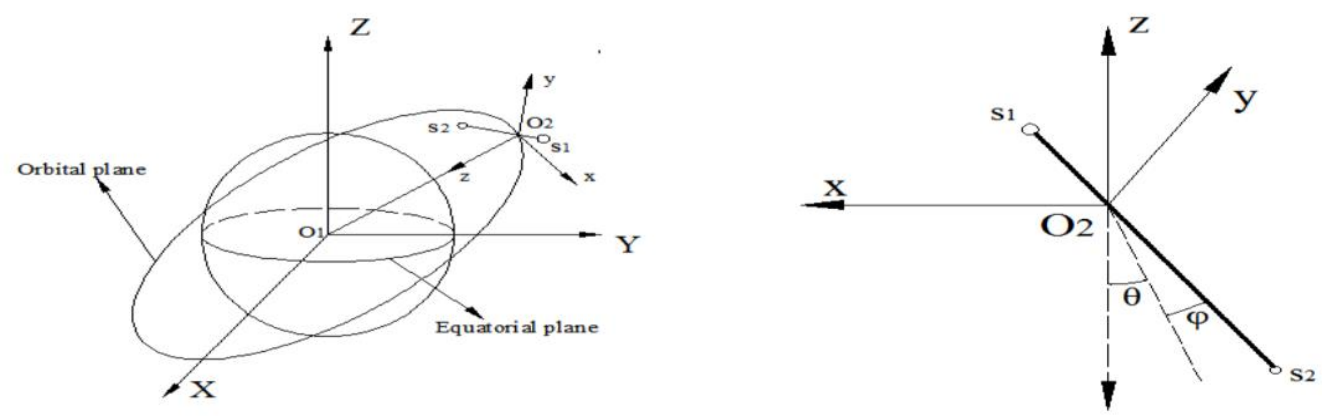

Figure 1. Simplified Tethered Satellite System Model 
It is assumed that the centre of mass of the TSS remains in a circular orbit. The state of TSS is represented by dimensionless vector $x=(\theta, \varphi, \Lambda, \dot{\theta}, \dot{\varphi}, \dot{\Lambda})$, which respectively represents in-plane libration angle, out-of-plane libration angle, length of the tether, in-plane angle velocity, out-of-plane angle velocity and tether motion velocity. Considering in-plane libration and out-of-plane libration motion, we built the dimensionless system dynamics equations of motion using the second Lagrange equation in spherical coordinates.

$$
\begin{aligned}
& \ddot{\theta}+2(\dot{\theta}-1)(-\dot{\varphi} \tan \varphi+\dot{\Lambda} / \Lambda)+3 \sin \theta \cos \theta=Q_{\theta} / \bar{m} \omega_{f}{ }^{2} L_{f}^{2} \cos ^{2} \varphi \\
& \ddot{\varphi}+2 \dot{\varphi} \dot{\Lambda} / \Lambda+\left[(\dot{\theta}-1)^{2}+3 \cos ^{2} \theta\right] \sin \varphi \cos \varphi=F_{t} \\
& \left.\ddot{\Lambda}-\Lambda\{\dot{(\varphi)})^{2}+\cos ^{2} \varphi\left[(\dot{\theta}-1)^{2}+3 \cos ^{2} \theta\right]-1\right\}=-u
\end{aligned}
$$

$m_{1}$ is the mass of the mother satellite, and $m_{2}$ is the mass of sub-satellite The former is greater than the latter, $m=m_{1}+m_{2}, \bar{m}=m_{1} m_{2} / m$ is the reduced system mass. $\Lambda=l(t) / L_{f}$ is the dimensionless length of the tether, $\boldsymbol{L}_{f}$ is reference length of the tether $\left(\boldsymbol{L}_{f}=1 \mathrm{Km}\right)$, the dimensionless time is expressed by $v=\Omega * t, \dot{\Lambda}=\left[d l(t) / L_{f}\right] *[d t / d v]=d l(t) /\left[\Omega * L_{f}\right]$ is the dimensionless time derivative. $Q_{\theta}$ is the generalized forces, $u=T / m_{2} \omega_{f}{ }^{2} L_{f}^{2}$ is the dimensionless tether tension, $T$ is the tether tension, $F=F_{t} / \bar{m} \omega_{f}{ }^{2} L_{f}^{2} \Lambda^{2}$ is the dimensionless thrust force, $F_{t}$ is the thrust force.

In this research, the hybrid control strategy was adopted to ensure that the sub-satellite is deployed to the target position accurately by controlling the tether tension and the thrust force in and out-of -plane.

\section{Nonlinear Receding Horizon Control}

The Receding horizon control (RHC) is an important method for feedback control for the complex systems. It is an optimization algorithm over a finite horizon. It forecasts the response and status of the controlled objects according to their prediction model and history information. In this research, the open-loop optimal solution is treated as the reference trajectory, and then the feedback control rule is designed on account of the nonlinear RHC method. The procedure for implementing the RHC for nonlinear problem could be summarized as follows. (1) The reference trajectory is obtained. (2) The quasilinearization is applied for nonlinear optimal problem, then Hamilton's equation is built and the optimal problem is transformed into the TPBVP solution problem. (3) Based on indirect pseudospectral method, the TPBVP problem is converted into a set of algebraic equations, then the linear equations are solved to obtain the current status and control input. (4) The time window is shifted continuously, and RHC technology is applied repeatedly until the maximum time is reached $[14,15]$. The control process is shown in Figure 2. 


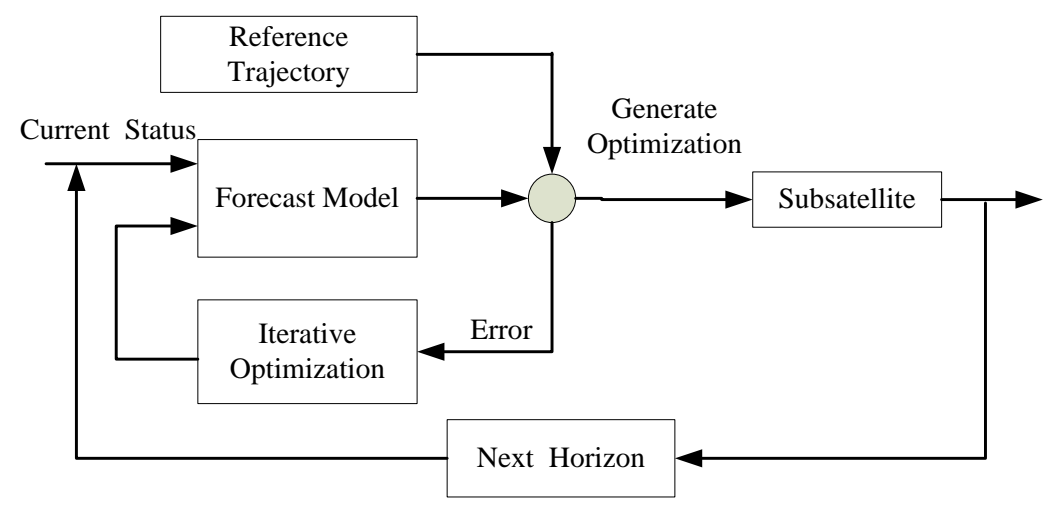

Figure 2. RHC Architecture Diagram

\subsection{Problem Formulation}

The optimal feedback control problem of RHC is to find the suitable perturbed state vectors $\delta x(t)$ and perturbed control vectors $\delta u(t)$, satisfying Eqs.(5)(6)(7), while minimizing the following performance indexes.

$$
\delta J=\frac{1}{2} \delta x^{T}(t+T) S_{f} \delta x(t+T)+\frac{1}{2} \int_{t}^{t+T}\left[\delta x^{T}(t) Q \delta x(t)+\delta u^{T}(t) R \delta u(t)\right] d v
$$

subject to the following constraints:

State equations: $\delta \dot{x}=f(\delta x(v), \delta u(v), v)=A \delta x(v)+B \delta u(v)$

Initial conditions: $\delta x(v=t)=\delta x(t)$

Terminal conditions: $\delta x(v=t+T)=0$

Where $\delta x(t)=x(t)-x_{\text {ref }}(t), \delta u(t)=u(t)-u_{\text {ref }}(t), \boldsymbol{x}_{\text {ref }}(t)$ and $\boldsymbol{u}_{\text {ref }}(t)$ are the reference state vectors and reference control vectors respectively. $\boldsymbol{A}$ represents the system state influence matrix. $\boldsymbol{B}$ represents the control influence matrix. $\boldsymbol{Q}$ is the positive semi-definite weighted matrix. $\boldsymbol{R}$ is defined as the positive definite weighted matrix. $\boldsymbol{S}$ is the positive semi-definite weighted matrix. $\boldsymbol{T}$ is the future horizon length. $\boldsymbol{t}$ is defined to be actual system time domain. $v$ is the prediction system time domain.

The nonlinear system can be solved via a quasilinearization method. The main idea of quasilinearization is to expand the performance index to be the second order, and to expand the system state equations to be first order around the norminal trajectory. Thus the nonlinear system could become the linear system around the norminal trajectory. The results of quasilinearization for Eqs. (5) are as follows

$$
\begin{aligned}
& \delta \dot{\tilde{x}}=\underset{\sim}{A}(v) \delta \tilde{x}+\underset{\sim}{B}(v) \delta \tilde{u}+\underset{\sim}{h}(v) \\
& \underset{\sim}{A}(v)=\left.\frac{\partial f(x(v), u(v), v)}{\partial x}\right|_{\underset{\sim}{x}(v), \underset{\sim}{u}(v)} \\
& \underset{\sim}{B}(v)=\left.\frac{\partial f(x(v), u(v), v)}{\partial u}\right|_{\underset{\sim}{x}(v), \underset{\sim}{u}(v)} \underset{\sim}{h}(v)=f(\underset{\sim}{x}(v), \underset{\sim}{u}(v), v)-\underset{\sim}{A}(v) \underset{\sim}{x}-\underset{\sim}{B}(v) \underset{\sim}{u}
\end{aligned}
$$


$\underset{\sim}{x}(v)$ and $\underset{\sim}{u}(v)$ represent state vectors and control vectors that the system iterated before thetime of $v$ respectively, while $\tilde{x}(v)$ and $\tilde{u}(v)$ represent state vectors and control vectors that the system iterated at the time of $v$ respectively.

The Hamiltonian for the system is given by

$$
H=\frac{1}{2}\left[\delta x^{T}(v) Q(v) \delta x(v)+\delta u^{T}(v) R(v) \delta u(v)\right]+\delta \tilde{\lambda}^{T}(v)[\underset{\sim}{A}(v) \delta x(v)+\underset{\sim}{B}(v) \delta u(v)+\underset{\sim}{h}(v)]
$$

From the Minimum Principle of Pontryagin, the necessary optimality condition yields the optimality condition:

$$
\frac{\partial H}{\partial \delta u}=0 \Rightarrow \delta \tilde{u}(v)=-R^{-1}(v) B^{T}(v) \delta \tilde{\lambda}(v)
$$

Where $\delta \lambda(v)$ is the co-state vectors and satisfies the Co-state equations

$$
\dot{\delta} \tilde{\lambda}(v)=-\frac{\partial H}{\partial \delta x}=-\left[Q(v) \delta \tilde{x}(v)+A_{\sim}^{T}(v) \delta \tilde{\lambda}(v)\right]
$$

Initial conditions: $\delta \tilde{x}(v=t)=\delta x(t)$

Terminal condition: $\delta \tilde{\lambda}\left(v_{f}=t+T\right)=S_{f}\left[\tilde{x}(t+T)-x_{f}\right]=S_{f} \delta \tilde{x}\left(v_{f}\right)$

$S$ satisfies the following Riccati differential equations:

$$
\begin{aligned}
& \dot{S}(v)=-S(v) A-A^{T} S(v)+S(v) B R^{-1} B^{T} S(v)-Q \\
& S_{f}=S(t+T)
\end{aligned}
$$

The sufficient conditions for the optimal solution of this method are as follows: Q must be a positive, semi-definite, weighted matrix, $R$ must be a positive definite weighted matrix, $S$ must be a positive semi-definite weighted matrix [16], and in the system equations $f(\underset{\sim}{x}(v), \underset{\sim}{u}(v), v)$ must be a continuously differentiable function. The LGL algorithm has the common problem that it is sensitive to initial conditions. Without proper initial conditions, the LGL method may fail to converge. If above the hypothesis conditions are satisfied, the proposed algorithm can achieve Nth order convergence.

The above state equations (8), the co-state equations (14) and the boundary conditions (15)(16) constitute a TPBVP. It has the following form:

$$
\left[\begin{array}{c}
\delta \dot{\tilde{x}} \\
\dot{\tilde{\tilde{\lambda}}}
\end{array}\right]=\left[\begin{array}{cc}
\underset{\sim}{A}(v) & -B(v) R^{-1}(v) B^{T}(v) \\
-Q(v) & -{\underset{\sim}{A}}^{T}(v)
\end{array}\right]\left[\begin{array}{c}
\delta \tilde{x} \\
\delta \tilde{\lambda}
\end{array}\right]+\left[\begin{array}{c}
h(v) \\
0
\end{array}\right]
$$

The traditional solution method for TPBVP problem relies on solving the time-varing Riccat equations via a backward sweep method or a transition matrix method, but these methods are potentially unstable. Backward sweep method relies on the explicit integration of the differential equations, which is both time consuming and numerically unstable. For illconditioned matrices, transition matrix methods can become very computationally expensive [17]. While the indirect pseudospectral method to solve TPBVP is based on a complete discretization of the states, the co-states and controls method is proposed and employed to provide feedback. Integrating the Riccati equations or inverting ill-conditioned transition matrix can be avoided [18,19]. The TPBVP problem (Eqs.19) can be discretized by the indirect Legendre pseudospectral method. In this approach, the state variables and co-state 
variables are expanded by Nth degree Polynomials, then the TPBVP is transformed into a set of algebraic equations which can be readily solved.

\section{2. Indirect LGL Pseudospectral Discretization}

Pseudospectral methods have many advantages including high precision operation, fast convergence rate, easy implementation. It has received more and more attention in the field of rocket launching and spacecraft trace control. Pseudospectral methods can be separated into direct pseudospectral methods and indirect pseudospectral methods. The main distinction between them is the different discrete objects. The discrete objects of the indirect pseudospectral method are two point boundary value problems constituted by system state equations and co-state equations, while the discrete objects of the direct pseudospectral method only refer to state equations. The indirect psuedospectral method is more sensitive to the initial guess and the convergence radius is smaller, but the solution is more accurate [14]. The direct pseudospectral method does not need to deduce the co-state equations. For most problems, direct method will obtain the solution with high computational efficiency, and it has a wider convergence radius. However, the solution does not contain the co-state information, which is not convenient for error analysis and optimality validation. So a combination of the direct and indirect methods can lead to a more accurate and efficient way of solving optimal control problems.

In this paper, nolinear optimal control problems are solved using the Legendre-GaussLobatto(LGL) pseudospectral method. The state vectors and control vectors are approximated through Nth degree Lagrange polynomial at LGL quadrature points. The differential constraints are transformed into algebraic equations using the differential matrix. The integral constraints are also transformed into algebraic equations by the Gauss-Lobatto integral rule $[20,21]$. The main procedure of the discretization for the optimal control problem by the LGL pseudospectral method is as follows.

The optimal control problem presented in the paper is formulated on the interval $\left[\boldsymbol{t}_{\boldsymbol{t}}, \boldsymbol{t}_{\boldsymbol{f}}\right]$, while in LGL pseudospectral method LGL points should be on the interval [-1,1]. So time domain should be transformed, and the affine transformation is as follows.

$$
t=\left[\left(t_{f}-t_{0}\right) \tau+\left(t_{f}+t_{0}\right)\right] / 2
$$

Using the above equation (4), LGL points are shifted from the computational domain $\tau \in\left[\tau_{0}, \tau_{f}\right]=[-1,1]$ to the physical domain $t \in\left[t_{0}, t_{f}\right]$.

The state and control functions, $\dot{x}(t)=f[x(t), u(t), t]$ are transformed into

$$
\dot{x}(\tau)=\frac{\left(t_{f}-t_{\mathrm{o}}\right)}{2} f[x(\tau), u(\tau), \tau]
$$

The state vectors and control vectors are approximated by LGL in forms:

$$
\begin{aligned}
& x(\tau) \approx x^{N}(\tau)=\sum_{k=0}^{N} x\left(\tau_{k}\right) \phi_{k}(\tau) \\
& u(\tau) \approx u^{N}(\tau)=\sum_{k=0}^{N} u\left(\tau_{k}\right) \phi_{k}(\tau)
\end{aligned}
$$

Where the basis functions 


$$
\phi_{k}(\tau)=\frac{\left(\tau^{2}-1\right) \dot{L}_{N}(\tau)}{\left(\tau-\tau_{K}\right) N(N+1)\left(L_{N}\left(\tau_{K}\right)\right)}
$$

are the lagrange interpolating polynomials, that interpolate the state vectors and control vector at the LGL points. $L_{N}(\tau)$ is the Nth degree Legendre interpolation polynomials on the interval $[-1,1] . \tau_{k}$ is LGL points, $\mathrm{k}=0, \ldots, \mathrm{N}$, which are given by $\tau_{0}=-1, \tau_{N}=-1$ and $\tau_{1}, \ldots$ $\tau_{N-1}$ are the zero points of $\dot{L}_{N}$.

Differential equation can be approximated at the LGL points, we approximate Eqs. (22)(23) and evaluate it at $\tau_{k}$ to obtain a matrix $\boldsymbol{D}_{\boldsymbol{k}, j}$ which results in:

$$
\dot{x}\left(\tau_{k}\right) \approx \dot{x}^{N}\left(\tau_{k}\right)=\xi \sum_{k=0}^{N} D_{k, j} x\left(\tau_{j}\right)
$$

$\xi$ is the transformation metric, it is determined as $\xi=d t / d \tau=\left(t_{f}-t_{0}\right) / 2$, where $D_{k, j}$ are entries of the $(N+1) \times(N+1)$ differential matrix. It is defined as:

$$
D_{k, j}=\left\{\begin{array}{cl}
\frac{L_{N}\left(\tau_{K}\right)}{L_{N}\left(\tau_{j}\right)} \frac{1}{\tau_{K}-\tau_{j}} & k \neq j \\
-\frac{N(N+1)}{4} & k=j=0 \\
\frac{N(N+1)}{4} & k=k=N \\
0 & \text { otherwise }
\end{array}\right.
$$

Eqs.(25) is substituted into Eq. (8)(14), State equations and Co-state equations can be expressed as:

$$
\begin{aligned}
& \frac{2}{T} \sum_{j=0}^{N} D_{k, j} \delta x\left(\tau_{j}\right)-A\left(\tau_{k}\right) \delta x\left(\tau_{k}\right)+B\left(\tau_{k}\right) R^{-1}\left(\tau_{k}\right) B^{T}\left(\tau_{k}\right) \delta \tilde{\lambda}\left(\tau_{k}\right)-h\left(\tau_{k}\right)=0 \\
& \frac{2}{T} \sum_{j=0}^{N} D_{k, j} \tilde{\lambda}\left(\tau_{j}\right)+Q\left(\tau_{k}\right) \delta \tilde{x}\left(\tau_{k}\right)+A^{T}\left(\tau_{k}\right) \tilde{\lambda}\left(\tau_{k}\right)=0 \\
& k=0, \ldots, N
\end{aligned}
$$

The integral in the performance index is approximated using the Gauss-Lobatto rule which provides a highly accurate result, and has the following form:

$$
\int_{t_{0}}^{t_{f}} f(t) d t=\frac{t_{f}-t_{0}}{2} \int_{-1}^{1} f(\tau) d \tau=\frac{t_{f}-t_{0}}{2} \sum_{k=0}^{N} w_{k} f\left(\tau_{k}\right)
$$

Where the above equation $w_{k}$ is the LGL integral weight, given by

$$
w_{k}=\frac{2}{N(N+1)} \bullet \frac{1}{\left[L_{N}\left(\tau_{K}\right)\right]^{2}} \quad K=0, \ldots, N
$$

Eqs. (29) is substituted into Eqs. (4), Performance index equations can be expressed as: 


$$
J=\frac{1}{2} \delta x^{T}\left(\tau_{N}\right) S_{f} \delta x\left(\tau_{N}\right)+\frac{T}{2} \sum_{k=0}^{N} w_{k}\left[\delta x^{T}\left(\tau_{k}\right) Q \delta x\left(\tau_{k}\right)+\delta u^{T}\left(t_{k}\right) R \delta u\left(\tau_{k}\right)\right]
$$

Boundary conditions discretization:

Initial condition: $\delta \tilde{x}\left(\tau_{0}\right)=\delta \tilde{x}(-1)=\delta x(t)$

Terminal condition: $\delta \tilde{\lambda}\left(\tau_{N}\right)=\delta \tilde{\lambda}(1)=S_{f} \delta \tilde{x}(1)=S_{f} \delta \tilde{x}\left(\tau_{N}\right)$

The vector of state variables and co-state variables is denote as

$$
X=\left[\delta x\left(\tau_{0}\right), \delta x\left(\tau_{1}\right), \ldots ., \delta x\left(\tau_{N}\right), \delta \lambda\left(\tau_{0}\right), \ldots, \delta \lambda\left(\tau_{N}\right)\right]^{T}
$$

The optimal problem is transformed into a set of algebraic equations

Where $\mathrm{A}$ is defined as

$$
\begin{gathered}
J=\frac{1}{2} X^{T} H X \\
A X=B
\end{gathered}
$$

$$
\begin{aligned}
& A=\left[\begin{array}{ll}
A^{11} & A^{12} \\
A^{21} & A^{22}
\end{array}\right] \\
& A_{k j}^{11}=\left\{\begin{array}{ll}
I & k=j=0 \\
0 & k=0, j \neq 0 \\
2 D_{k, j} / T & k \neq j, k \neq 0 \\
2 D_{k, j} / T-\underset{\sim}{A}\left(\tau_{k}\right) & k=j \neq 0
\end{array} \quad A_{k j}^{12}= \begin{cases}\underset{\sim}{B}\left(\tau_{k}\right) R^{-1}\left(\tau_{k}\right) \underset{\sim}{B^{T}}\left(\tau_{k}\right) k=j \neq 0 \\
0 & \text { otherwise }\end{cases} \right. \\
& A_{k j}^{21}=\left\{\begin{array}{ll}
Q\left(\tau_{k}\right) & k=j \neq N \\
0 & k \neq j \\
-S_{f} & k=j=N
\end{array} \quad A_{k j}^{22}= \begin{cases}2 D_{k, j} / T & k \neq j, k \neq N \\
2 D_{k, j} / T+A_{\sim}\left(\tau_{k}\right) & k=j \neq N \\
0 & k=N, j \neq N \\
I & k=j=N\end{cases} \right. \\
& B=\left[x(t), \underset{\sim}{h}\left(\tau_{1}\right), \ldots ., \underset{\sim}{h}\left(\tau_{N}\right), 0,0, \ldots, 0\right]^{T}
\end{aligned}
$$

$\boldsymbol{I}$ refers to the unit vector of $\boldsymbol{n} \times \boldsymbol{n}, 0$ represents the zero vector of $\boldsymbol{n} \times \boldsymbol{n}$. The first equation of the system above expresses the initial condition constraints. The last equation expresses the terminal condition constraints.

\subsection{Feedback Control Gain:}

The nonlinear optimal control problem of TSS is discretized and transformed to a nonlinear programming problem by the LGL pseudospectral method is detailed above. Then the open-loop optimal control law may be obtained by solving the nonlinear programming problem. The open-loop control law only depends on the input variables which change over time, neglecting the effect of model errors and external disturbances, So the open-loop trajectories for state and control vectors are only obtained under ideal conditions. To counteract the influence of the above factors, the closed-loop control uses the feedback information to compensate for the deviations in real time which is occurring during the operation of system. 
After the system is discretized, $S\left(\tau_{k}\right)$ can be obtained according to the following equations,

$$
\frac{2}{T} \sum_{j=0}^{N} D_{k, j} S\left(\tau_{j}\right)=-S\left(\tau_{k}\right) A-A^{T} S\left(\tau_{k}\right)+S\left(\tau_{k}\right) B R^{-1} B^{T} S\left(\tau_{k}\right)-Q
$$

So at the LGL points, the closed-loop feedback gains of the system can be expressed as:

$$
K(\tau ; N, T)=-R^{-1}\left(\tau_{k}\right) B^{T}\left(\tau_{k}\right) S\left(\tau_{k}\right)
$$

$K$ is the time-varying feedback gain matrix which can be solved in real time. $K(\tau ; N, T)$ can be expressed as $k\left(\tau_{k}\right)$.where $\tau_{k} \in[-1,1], \boldsymbol{k}=0, \ldots, N$

The optimal closed-loop control of the system could be shown as follows:

$$
\begin{gathered}
\frac{2}{T} \sum_{j=0}^{N} D_{k, j} S\left(\tau_{j}\right)=-S\left(\tau_{k}\right) A-A^{T} S\left(\tau_{k}\right)+S\left(\tau_{k}\right) B R^{-1} B^{T} S\left(\tau_{k}\right)-Q \\
\delta \tilde{u}\left(\tau_{k}\right)=-R^{-1}\left(\tau_{k}\right) B^{T}\left(\tau_{k}\right) S\left(\tau_{k}\right) \delta \tilde{x}\left(\tau_{k}\right)=K\left(\tau_{k}\right) \delta x\left(\tau_{k}\right)
\end{gathered}
$$

Except the LGL points, other values can be obtained via interpolation

\section{Numerical Example}

For this numerical example, there are several constraints. The tether tension should be greater than zero to avoid the possibility of the tether becoming tangled du to the slack meanwhile the tension should not be great enough to snap the tether. The in-plane libration angle and the angle out-of-plane libration angle should not change too greatly in order to avoid interfering with the mother satellite. The terminal constraints demand that the tether should reach the specified target. They also demand the velocity of in-plane libration angle and out-of-plane libration angle are close to zero and the tether tension should remain positive throughout the deployment process.

All the vectors are transformed into dimensionless vectors. The dimensionless deployment length of tether may vary in the interval $\left[\boldsymbol{L}_{\min }, \boldsymbol{L}_{\max }\right]$, where $\boldsymbol{L}_{\min } \geqq 0.1, \boldsymbol{L}_{\max } \leqq 1$, the initial value of tether length is 0.1 .The dimensionless tether tension may vary in the interval $\left[T_{\min }\right.$, $T_{\max }$ ], where $\boldsymbol{T}_{\min } \geqq 0.01, \boldsymbol{T}_{\max } \leqq 6 . \boldsymbol{t}_{\boldsymbol{0}}$ is the initial time, and $\boldsymbol{t}_{\boldsymbol{f}}$ is the terminal time. Only 40 LGL points are used in order to keep the computational cost low, since this is intended to be run in real time. The error tolerances of the function iteration are set to 1e-7.

(1) Case1: open-loop control with no disturbance

Initial constraints: $x\left(t=t_{0}\right)=[\theta, \dot{\theta}, \dot{\varphi}, \dot{\varphi}, \Lambda \dot{\Lambda}]=[0,0,0,0,0.1, \mathbf{0}]$

Terminal constraints: $x\left(t=t_{f}\right)=[\theta, \dot{\theta}, \dot{\varphi}, \dot{\varphi}, \Lambda, \dot{\Lambda}]=[0,0,0,0,1, \boldsymbol{0}]$.

(2) Case2: Closed-loop control with a initial disturbance

Initial constraints: $\boldsymbol{x}\left(\boldsymbol{t}=t_{0}\right)=[\theta, \dot{\theta}, \dot{\varphi}, \dot{\varphi}, \Lambda, \dot{\Lambda}]=[0.1,0.1,0.05,0,0.2,0.1]$;

Terminal constraints: $x\left(t=t_{f}\right)=[\theta, \dot{\theta}, \dot{\varphi}, \dot{\varphi}, \Lambda, \dot{\Lambda}]=[0,0,0,0,1,0]$.

Weight matrix: $\boldsymbol{S}_{f}=\operatorname{diag}[1,1,1,1,10,1], \boldsymbol{Q}=\operatorname{diag}[1,1,10,100,1,1] ; \boldsymbol{R}=\operatorname{diag}[1,0.02]$;

The Performance index could make the system energy to be optimal and make the system change smoothly. 
Figure 3 shows the time history of the dimensionless control input under open-loop optimal and closed-loop optimal control with a initial disturbance.
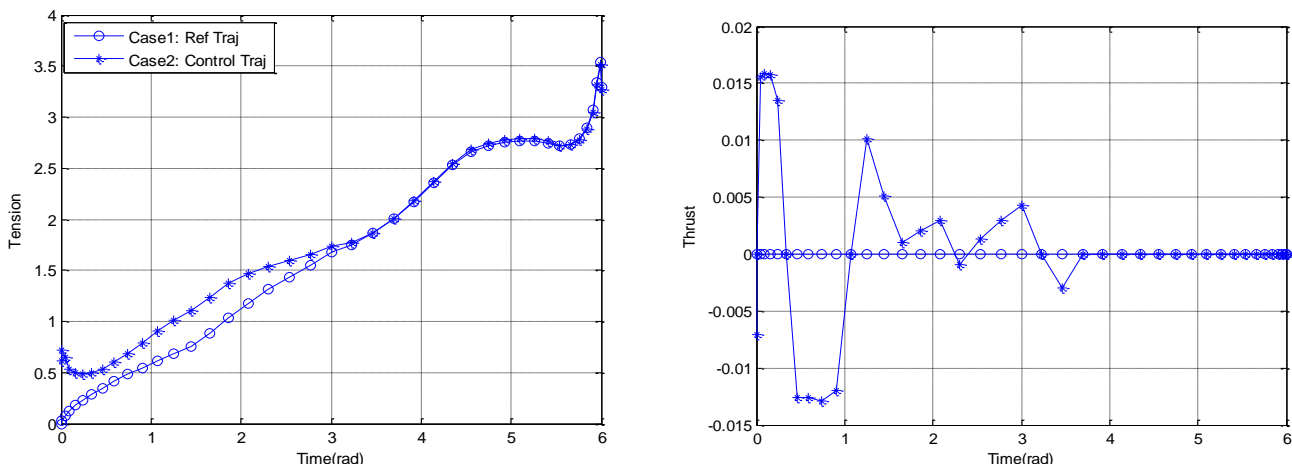

Figure 3. The Optimal Values of the Dimensionless Control Input

Figure 3 indicates that the control input mainly depends on the tether tension, which is controlled accurately and is smooth, while the thrust force is small. This hybrid control method could result in considerable energy savings. At the same time, it could also reduce the complexity of designing the subsatellite structure.

Figure 4 shows the time history of the dimensionless state variables under open-loop optimal and closed-loop optimal control. The dimensionless states include in-plane libration angle, out-of-plane libration angle, length of the tether, in-plane angle velocity, out-of-plane angle velocity and tether motion velocity.
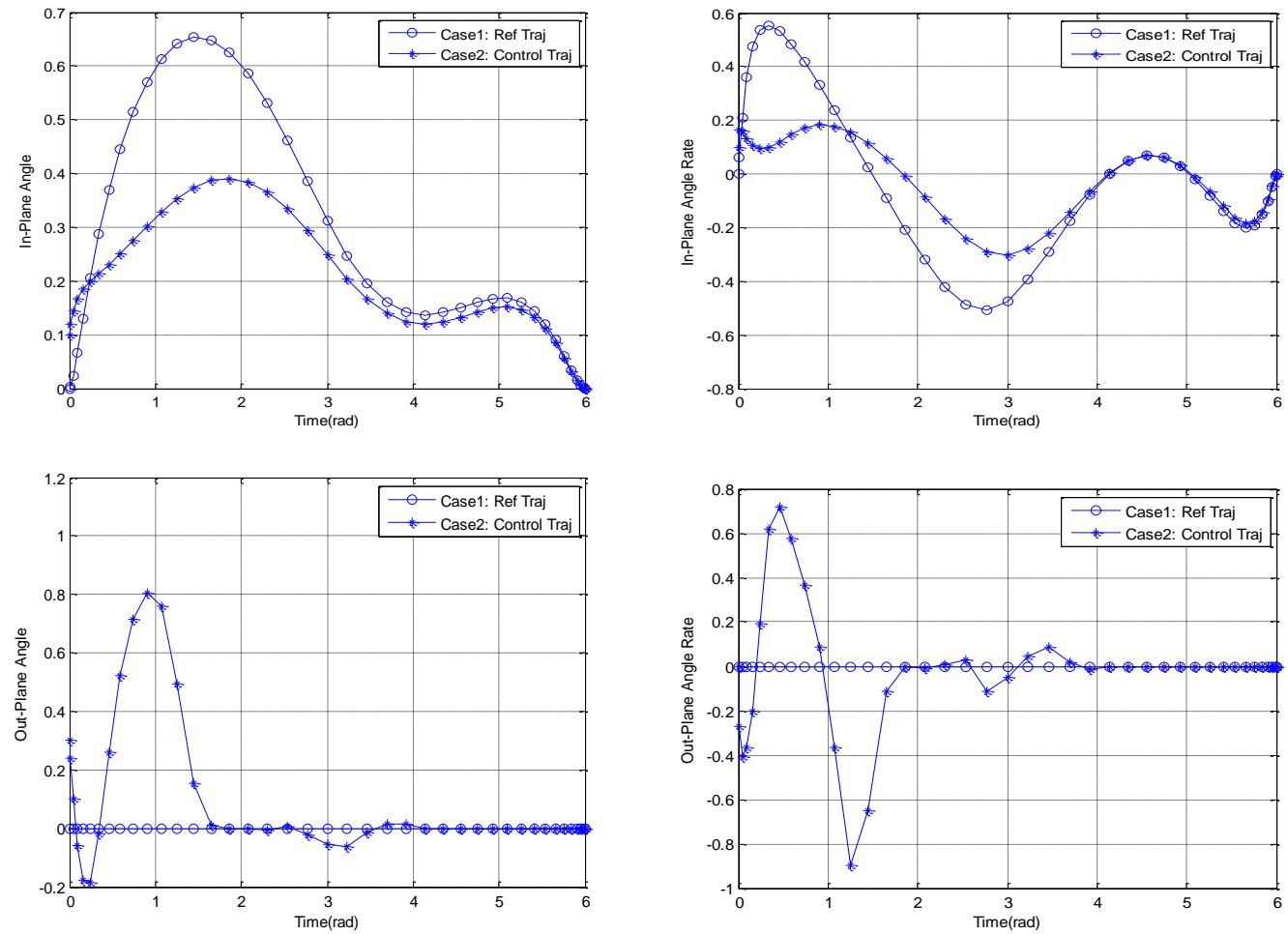

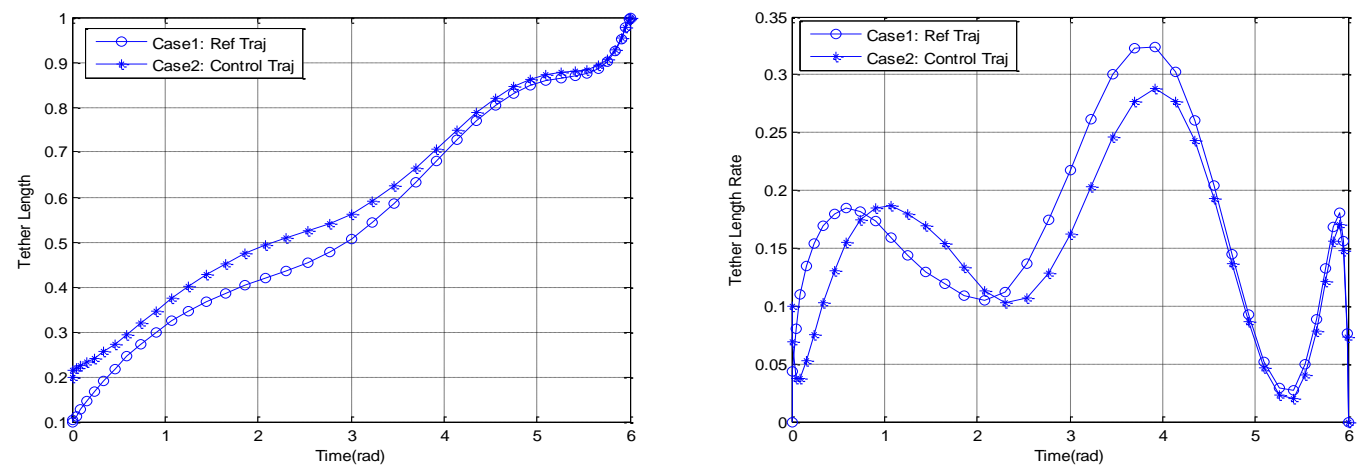

Figure 4. The Optimal Value of the Dimensionless State Variables

Under open-loop control in case 1, the state variables changed slightly, and out-of-plane angle and the velocities of the out-plane angles were always closed to zero. Under feedback control in case 2, the effect of disturbance is added in the initial states. The differences of the operation curve between case 1 and case 2 are obvious in the initial stage. After the feedback control was compensated momentarily, the state variables of the open-loop and closed-loop converged. So RHC can mitigate the influence of disturbances efficiently to some extent, and it can also keep the system close to the reference trajectory. These results indicate that the trajectory converges to the reference after several sampling times.

\section{Conclusions}

The new method for solving the nonlinear RHC problem in the deployment of tethered satellites using an indirect pseudospectral method is presented. The control algorithm retains the accuracy of an indirect pseudospectral method, and obtains the value of co-state vector, while reducing the computation time and improving the computational efficiency by transforming the TPBVP problem into a set of linear algebraic equations. The efficiency of the method makes if valid for real time operation. Simulation results show that the system can remain stable and accurate even with initial disturbances. The controller can ensure that the subsatellite is depolymented to the destination accurately.

\section{Acknowledgements}

This work is supported by the National Equipment Pre-research Fund of China under Grant 62501040402.

\section{References}

[1] M. L. Cosmo, E. C. Lorenzini, "Tethers In Space Handbook, 3rd ed. Smithsonian Astrophysical Observatory", Cambridge MA, (1997).

[2] J. A. Carroll, "Tether Applications in Space Transportation", Acta Astronautica, vol. 13, no. 4, (1984), pp. 165-174.

[3] M. P. Cartmell, D. J. McKenzie, “A review of sp"ace tether research”, Progress in Aerospace Sciences”, vol. 44, no. 1, (2008), pp. 1-21.

[4] K. D. Kumar, "Review of dynamics and control of nonelectrodynamic tethered satellite systems", Journal of Spacecraft and Rockets, vol. 43, no. 4, (2006), pp. $705-720$.

[5] G.Rega, "Nonlinear vibrations of suspended cables - Part I: Modeling and analysis", Applied Mechanics Reviews, Vol. 57, no. 6, (2004), pp. 443-478.

[6] A. Steindl, H. Troger, "Optimal control of deployment of a tethered subsatellite", Nonlinear Dynamics, Vol. 31, no. 3, (2003), pp.257-274. 
[7] H. Glel, F. Zimmermann, S. Brückner, "Adaptive neural control of the deployment procedure for tetherassisted re-entry”, Aerospace Science and Technology, vol. 8, no. 1, (2004), pp. 73-81.

[8] P. Williams, A. Hyslop, M. Stelzer, M. Kruijff, "YES2 optimal trajectories in presence of eccentricity and aerodynamic drag”, Acta Astronautica, vol. 64, no. 7, (2009), pp. 745-769.

[9] H. Yan, F. Rahroo, I. M. Ross, "Optimal Feedback Control Laws by Legendre Pseudospectral Approximations", Proceedings of the American Control Conference, (2001), June 25-27; Arlington,VA, American.

[10] T. Ohtsuka, "Time-variant receding horizon control of nonlinear system", Journal of Guidance, Control, and Dynamics, vol. 21, no. 1, (1998), pp. 174-176.

[11] P. Lu, "Regulation about Time-Varying Trajectories: Precision Entry Guidance Illustrated", Journal of Guidance, Control, and Dynamics, vol. 22, no. 6, (1999), pp. 784-790.

[12] D. P. Jin, H. Y. Hu, "Optimal control of a tethered subsatellite of three degrees of freedom", Nonlinear Dynamics, vol. 46, no. 1, (2006), pp. 161-178.

[13] H. Wen, D. P. Jin, H. Y. Hu, "Optimal feedback control of the deployment of a tethered subsatellite subject to perturbations", Nonlinear Dynamics, vol. 51, no. 4, (2008), pp. 501-514.

[14] P. Williams, C.Blanksby, P.Trivailo, H. A. Fujii, "Receding Horizon Control Of Tethers System Using Quasilinearisation and Chebyshev Pseudospectral Approximations", Advances in the Astronautical Sciences, vol. 116, no. 1, (2003), pp. 539-558.

[15] P. Williams, "Application of Pseudospectral Methods for Receding Horizon Control”, Journal of Guidance, Control, and Dynamics, vol. 27, no. 2, (2004), pp. 310-314.

[16] X. Xu and S. K. Agrawal, "Finite-Time Optimal Control of Polynomial Systems Using Successive Suboptimal Approximations", Journal of Optimization Theory and Applications, vol. 105, no. 2, (2000), pp. 477-489.

[17] C. F. Gerald, P. O. Wheatley, “Applied Numerical Analysis”, Addison-Wesley Publishing Company, (1984).

[18] F. Fahroo, I. M. Ross, "Trajectory optimization by indirect spectral collocation methods", Proceedings of the AIAA/AAS Astrodynamics specialist conference, (2000); Denver.

[19] H. Yan, F. Fahroo, I. M. Ross, "Optimal Feedback Control Laws by Legendre Pseudospectral Approximations", Proceedings of the American Control Conference , (2001), June 25-27; Arlington, American.

[20] F. Fahroo, I. M. Ross, "Co-state estimation by a Legendre pseudospectral method", Journal of Guidance, Control, and Dynamics, vol. 24, no. 2, (2001), pp. 270-277.

[21] I. M. Ross, F. Fahroo, "Legendre pseudospectral approximations of optimal control problems, New Trends in Nonlinear Dynamics and Control, and Their Applications", Lecture Notes in Control and Information Sciences, vol. 295, (2003), pp. 327-342.

\section{Authors}

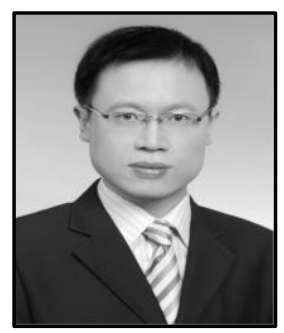

Guoyong Li, he is currently pursuing the Ph.D. degree of mechatronic engineering at School of Mechanical Engineering, Shandong University. He received the M.S. degree from the school of automation, Beijing University of posts and telecommunications. His research interests includes nonlinear control, optimal control of spacecraft. He has also been worked in National Network Software Testing Center (Jinan).

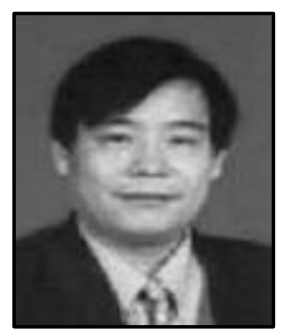

Changhou Lu, he is currently a professor and a doctoral supervisor of mechatronic engineering at School of Mechanical Engineering of Shandong University. He has published over 120 papers on vibration control, trace control and high-speed mechanical control. He has successfully completed over 20 research projects supported by national and ministry funds. 


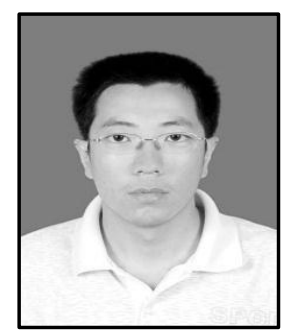

Wei Pan, he is currently a Lecturer of mechatronic engineering at School of Mechanical Engineering, Shandong University. He received the M.S. and Ph.D. degrees from Shandong University, both in mechatronic engineering, in 2001 and 2010, respectively. His research interest includes optimal control, nonlinear programming, low-thrust orbital maneuvers, cooperative control of autonomous vehicles, space maneuvers for tethered systems and industry applications of control theory.

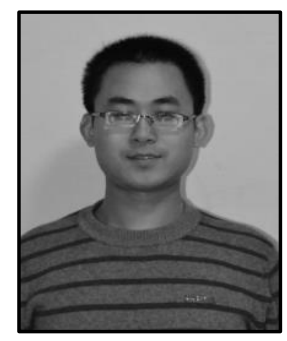

Qing Wang, he is currently pursuing the Ph.D. degree of mechatronic engineering at School of Mechanical Engineering, Shandong University. He received the B.E. degree in manufacturing science and engineering from Sichuan University, Sichuan,China, in 2011. His research interests included nonlinear control system, adaptive control, and distributed parameter control system. 
International Journal of Control and Automation Vol.7, No.9 (2014) 